\title{
Seleção Adaptativa de Operadores Aplicada ao Problema do Despacho Econômico de Energia Elétrica
}

\author{
Richard Aderbal Gonçalves ${ }^{1}$, Carolina Paula de Almeida ${ }^{1}$, \\ Sandra Mara G. S. Venske ${ }^{1}$, Josiel N. Kuk ${ }^{1}$, Lucas M. Pavelski ${ }^{1}$ \\ ${ }^{1}$ Departamento de Ciência da Computação - Universidade Estadual do Centro-Oeste \\ UNICENTRO - Guarapuava - PR - Brasil \\ \{richard, carol, ssvenske, jnkuk, lmpavelski\}@unicentro.br
}

\begin{abstract}
The Economic Load Dispatch Problem is one of the most important problems in the generation and distribution of electrical energy. Differential Evolution is an efficient evolutionary algorithm for continuous optimization. Distinct Differential Evolution operators are adequate to solve problems with distinct characteristics, however the choice of which operator is more adequate is a complex task. In this work, two adaptive operator selection techniques (Adaptive Pursuit and Probability Matching) are investigated to select the most appropriate operator to solve the Economic Load Dispatch Problem during execution time. The proposed algorithms are evaluated in problems with 13 and 40 thermal generators with valve-point loading effect. The proposed methods achieved better results than recent metaheuristics, finding the best known minimum cost value for all considered systems.
\end{abstract}

Resumo. O Despacho Econômico de Energia Elétrica é um dos mais importantes problemas na área de geração e distribuição de energia elétrica. A Evolução Diferencial é um algoritmo evolutivo eficiente para otimização contínua. Diferentes operadores da Evolução Diferencial são adequados para a resolução de problemas com características diferentes, contudo a escolha do operador mais adequado é uma tarefa complexa. Neste trabalho são investigadas duas técnicas de seleção adaptativa de operadores (Adaptive Pursuit $e$ Probability Matching) para escolher em tempo de execução qual o operador mais eficiente para a resolução do Despacho Econômico de Energia Elétrica. Os algoritmos propostos são validados em problemas de teste que consideram 13 e 40 geradores térmicos e levam em consideração efeitos de ponto de válvula. Os métodos propostos superam os resultados reportados na literatura obtidos por metaheurísticas modernas, sendo capazes de encontrar o melhor valor de custo mínimo conhecido para todos os sistemas de teste considerados.

\section{Introdução}

O Despacho Econômico de Energia Elétrica (DEEL) é um dos mais importantes problemas a serem resolvidos durante o planejamento e operacionalização de um sistema de geração de energia elétrica [Park et al. 2006]. O objetivo do despacho econômico é minimizar o custo total de combustível de geradores de energia elétrica sujeito a restrições operacionais. Neste trabalho, são considerados os efeitos de ponto de válvula na geração de energia, tornando o problema de otimização não-linear e multimodal. O DEEL na 
prática corresponde a um problema de otimização complexo no qual o uso de técnicas de otimização clássicas mostram-se ineficientes e, portanto, técnicas de inteligência computacional [Engelbrecht 2007], têm se tornado populares na sua solução.

Neste trabalho é explorada a técnica de Evolução Diferencial (ED)[Storn and Price 1997]. Diferentes operadores da ED se mostram mais eficientes de acordo com as características dos problemas a serem resolvidos. A determinação do operador mais adequado é uma tarefa árdua e, além disso, a adequação dos operadores pode depender do estado atual do processo de otimização, ou seja, diferentes operadores podem ser mais eficientes em diferentes etapas da otimização. A seleção adaptativa de operadores visa determinar em tempo de execução qual ou quais operadores devem ser utilizados em cada etapa do processo de otimização de maneira automática [Gong et al. 2011]. Duas das principais técnicas de seleção adaptativa de operadores são a Adaptive Pursuit (AP) e Probability Matching (PM) [Thierens 2005]. Portanto, esse trabalho visa investigar a adequação do uso da AP e da PM para a seleção de operadores da ED para a resolução do Problema do Despacho Econômico de Energia Elétrica.

O restante deste trabalho é organizado conforme a seguir. Na Seção 2 o problema do Despacho Econômico de Energia Elétrica é brevemente descrito. A Seção 3 apresenta os principais conceitos da Evolução Diferencial e de seleção adaptativa de operadores. Os métodos propostos são descritos na Seção 4 enquanto a Seção 5 apresenta os experimentos e os resultados obtidos com os algoritmos implementados, comparando-os com algoritmos da literatura. Finalmente, a Seção 6 contém as conclusões e trabalhos futuros.

\section{Despacho Econômico de Energia Elétrica}

Este trabalho foca na otimização da geração de energia elétrica, ou seja, na minização dos custos de produção de energia. Tal otimização tem grande importância econômica na sociedade moderna.

Uma usina elétrica possui vários geradores, sendo cada gerador responsável por produzir parte da energia demandada $\left(P_{D}\right)$. Cada gerador tem atributos diferentes e para que se tenha a melhor eficiência de todos os geradores, deve-se ajustar os parâmetros de geração de energia de cada gerador, de modo que o custo conjunto de todos seja minimizado. O problema de despacho de energia elétrica pode ser descrito de maneira formal do seguinte modo [Basu 2002]:

$$
\begin{gathered}
\text { Minimizar } \mathrm{C}=\sum_{j=1}^{n} C_{j}\left(P_{j}\right) \\
\text { sujeito a } \sum_{j=1}^{n} P_{j}-P_{D}=0 \quad \mathrm{e} \\
P_{j}^{\text {min }} \leq P_{j} \leq P_{j}^{\text {max }}
\end{gathered}
$$

tal que, C é o custo total considerando todos os geradores, $P_{j}$ é a energia produzida pelo j-ésimo gerador, $C_{j}\left(P_{j}\right)$ é a função de custo do j-ésimo gerador (em $\$ / \mathrm{hr}$ ), $P_{j}$ é a potência de saída da $j$-ésima unidade, $n$ é o número de unidades geradoras no sistema, $P_{D}$ é a demanda total de potência, $P_{j}^{\min }$ e $P_{j}^{\max }$ são, respectivamente, a potência de saída mínima e máxima da j-ésima unidade. A restrição de igualdade da Equação 1 é chamada de restrição de balanceamento de potência enquanto a restrição de desigualdade é chamada de restrição operacional. De acordo com as restrições, a potência total gerada deve ser igual a demanda total do sistema e a potência gerada por cada unidade deve estar 
entre os seus limites mínimos e máximos.

O cálculo de custo (C) é dado pela soma do custo de cada gerador. Neste trabalho, o custo dos geradores leva em consideração o efeito de ponto de válvula, que é a interferência no custo por conta da abertura de válvulas [Basu 2002]. Considerando o efeito de ponto de válvula, o custo de cada gerador é afetado por uma função senoidal, conforme a Equação 2:

$$
\begin{gathered}
C_{j}\left(P_{j}\right)=a_{j} * P_{j}^{2}+b_{j} * P_{j}+c_{j}+ \\
\left|e_{j} * \sin \left(f_{j} *\left(P_{j}^{m i n}-P_{j}\right)\right)\right|
\end{gathered}
$$

tal que $a_{j}, b_{j}, c_{j}, e_{j}$ e $f_{j}$ são os coeficiente empíricos de custo de combustível da j-ésima unidade com efeito de ponto de válvula.

\section{Evolução Diferencial: clássica e adaptativa}

A Evolução Diferencial (ED) [Storn and Price 1997] é um algoritmo evolutivo para otimização numérica que, basicamente, adiciona a diferença ponderada entre dois vetores da população (chamados de vetores de diferença) a um terceiro vetor (chamado de vetor alvo). A ED trabalha com alguns parâmetros como tamanho da população $(N)$, constante de cruzamento $(C R)$ e peso do vetor de diferenças - constante de mutação $(F)$.

O algoritmo da ED possui algumas variações, conhecidas como estratégias de mutação. A notação adotada para caracterizar as variações é $\mathrm{ED} / x / y / z$. Estas estratégias diferem especialmente na forma em que os vetores utilizados no cálculo das diferenças são escolhidos $(x)$, no número de vetores de diferença utilizados $(y)$ e na forma em que o ponto de cruzamento é determinado ( $z$ ). Neste trabalho são utilizadas as estratégias ED/rand/1/bin, ED/rand/2/bin, ED/current-to-rand/1 e ED/current-to-rand/2. Basicamente, indivíduos aleatórios (rand) ou o indivíduo corrente e outros aleatórios (currentto-rand) são selecionados, há $y=1$ ou $y=2$ pares de soluções que são aleatoriamente escolhidas para calcular a mutação diferencial e o cruzamento utilizado é o binomial $(z=b i n)$ ou específico (ED/current-to-rand/1 e ED/current-to-rand/2) [Storn and Price 1997].

O desempenho do algoritmo de evolução diferencial é altamente dependente da escolha dos seus parâmetros. O método de "tentativa e erro" para ajustar os parâmetros e escolher a melhor estratégia de mutação a ser utilizada requer tempo e conhecimento tanto sobre a técnica quanto sobre o problema abordado. Na literatura, há alguns algoritmos com controle adaptativo de parâmetros para a ED, tais como: SaDE [Qin and Suganthan 2005, Qin et al. 2009], JADE [Zhang and Sanderson 2009], EPSDE [Mallipeddi et al. 2011], AdapSS [Gong et al. 2010, Gong et al. 2011], AdapMODE [Li et al. 2011], ENS-MOEA/D [Zhao et al. 2012], entre outros. Algumas destas abordagens utilizam o conceito de conjunto (ou pool) de estratégias. Nestre trabalho, a parte adaptativa da ED adota os métodos de Probability Matching (PM) e Adaptive Pursuit (AP) [Gong et al. 2011, Thierens 2005, Goldberg 1990] para atualizar a probabilidade de aplicação de cada uma das quatro estratégias de mutação da ED adotadas.

Considerando $S>1$ estratégias no conjunto de operadores $\varsigma=\left\{s_{1}, \cdots, s_{S}\right\}$ e o vetor de probabilidade $\mathbf{p}(g)=\left(p_{1}(g), \cdots, p_{S}(g)\right)$, sendo $p_{s}(g)$ a probabilidade de uso da $s$-ésima estratégia na geração $g, \sum_{s=1}^{S} p_{s}(g)=1 ; \forall g: p_{\min } \leq p_{s}(g) \leq 1$, sendo que $p_{\min }$ é o valor de probabilidade mínimo de cada estratégia, utilizado para garantir 
que não se perca nenhuma estratégia [Thierens 2005]. Há muitos métodos para atualizar adaptativamente a probabilidade $p_{s}(g)$ da s-ésima estratégia, geralmente baseados na sua qualidade estimada empiricamente $q_{s}(g)$. Na maior parte do tempo, $q_{s}(g)$ é atualizada por um valor de recompensa recebido. Denota-se $r_{s}(g)$ como a recompensa que a $s$ ésima estratégia recebe depois de ser adotada na geração $g$. A recompensa adotada nesse trabalho é dada pela Equação 3.

$$
r_{s}(g)=\text { fitness }_{\text {melhor }} / \text { fitness }_{\text {filho }} * \mid \text { fitness }_{\text {pai }}-\text { fitness }_{\text {filho }} \mid
$$

A qualidade estimada empiricamente $q_{s}(g)$ da s-ésima estratégia na geração $g$ pode ser atualizada como a seguir [Thierens 2005]:

$$
q_{s}(g+1)=q_{s}(g)+\alpha *\left[r_{s}(g)-q_{s}(g)\right]
$$

tal que $\alpha \in(0,1]$ é a taxa de adaptação. Quanto maior a taxa de adaptação, maior a contribuição das recompensas mais recentes.

Com base nesta medida estimada, PM e AP diferem na forma em que estes métodos utilizam esta informação $\left(q_{s}(g+1)\right)$ a fim de atualizar a probabilidade do uso de cada estratégia. O método Probability Matching calcula $p_{s}(g+1)$ como a seguir [Gong et al. 2011, Thierens 2005, Goldberg 1990]:

$$
p_{s}(g+1)=p_{\text {min }}+\left(1-S * p_{\text {min }}\right) * \frac{q_{s}(g+1)}{\sum_{s=1}^{S} q_{s}(g+1)}
$$

Claramente, $\sum_{s=1}^{S} p_{s}(g+1)=1$. A partir da Eq. 5 pode ser notado que quando somente uma estratégia obtém valor de recompensa durante um longo período de tempo, sua probabilidade de seleção converge para $p_{\max }=p_{\min }+\left(1-S * p_{\min }\right)$.

O método Adaptive Pursuit foi inicialmente projetado para seleção adaptativa de operadores no contexto de Algoritmos Genéticos [Thierens 2005]. AP calcula $p_{s}(g+1)$ com a seguir:

$$
p_{s^{*}}(g+1)=p_{s^{*}}(g)+\beta *\left[p_{\max }-p_{s^{*}}(g)\right]
$$

$\mathrm{e} \forall s \neq s^{*}: p_{s}(g+1)=p_{s}(g)+\beta *\left[p_{\min }-p_{s}(g)\right], \operatorname{com} s^{*}=\operatorname{argmax}_{s}\left(q_{s}(g+1)\right)$ e $p_{\max }=$ $p_{\text {min }}+1-S * p_{\text {min }}$.

Esta restrição garante que se $\sum_{s=1}^{S} p_{s}(g)=1$, então $\sum_{s=1}^{S} p_{s}(g+1)=1$ [Thierens 2005]. O método AP tem uma taxa de aprendizado $\beta \in(0,1]$, que controla quão gulosa será a estratégia adaptativa.

\section{Algoritmos Propostos - $\mathrm{ED}_{A P}$ e ED $\mathrm{ED}_{P M}$}

Neste trabalho são propostas duas variações da Evolução Diferencial utilizando seleção adaptativa de operadores: $\mathrm{ED}_{A P}$ e $\mathrm{ED}_{P M}$. A diferença entre ambas propostas reside somente na técnica de seleção e, portanto, o Algoritmo 1 descreve os passos tanto do $\mathrm{ED}_{A P}$ quanto do $\mathrm{ED}_{P M}$.

O primeiro passo do algoritmo consiste na inicialização aleatória das soluções, respeitando as restrições operacionais de cada gerador. Como os indivíduos da população 
inicial podem não respeitar a restrição de demanda, o procedimento de reparação é chamado (ver Algoritmo 2). A seguir o custo de todas as soluções é calculado, conforme as Equações 1 e 2. Na sequência o algoritmo entra em seu laço principal, o qual é repetido até que o critério de parada seja satisfeito (neste trabalho corresponde a um número máximo de avaliações). No laço principal cada solução passa pelas etapas de seleção e aplicação dos operadores, avaliação e reparação das soluções geradas, cálculo das recompensas e das qualidades empíricas, bem como a atualização das probabilidades de aplicação de cada operador.

A seleção do operador da ED a ser aplicado é estocástica, dependendo apenas da probabilidade atual de aplicação de cada operador (inicialmente todos os operadores possuem a mesma probabilidade). De acordo com a operação selecionada são escolhidos $\mathrm{M}$ indivíduos para a aplicação do operador. $\mathrm{O}$ valor de $\mathrm{M}$ é 3 para os operadores $\mathrm{ED} / \mathrm{rand} / 1 /$ bin e ED/current-to-rand/1 e 5 para os operadores ED/rand/2/bin e ED/currentto-rand/2. Finalmente, o operador da Evolução Diferencial é aplicado para a geração de uma nova solução.

A nova solução pode ser infactível e, portanto, deve ser reparada através do procedimento descrito no Algoritmo 2. Após a reparação a nova solução é avaliada e a seleção é aplicada. No caso da ED a seleção é um torneio entre a solução original e a nova solução gerada, onde a melhor é mantida na população.

Os cálculos da recompensa e da qualidade empírica do operador utilizado são feitos de acordo com as Equações 3 e 4, respectivamente. Finalmente, no último passo do laço principal, as probabilidades de aplicação de cada operador são atualizadas de acordo com a Equação 5 caso a técnica Probability Matching esteja sendo utilizada ou pela Equação 6 caso a Adaptive Pursuit esteja sendo empregada.

\section{Resultados}

Foram realizadas 50 execuções independentes de cada algoritmo nos três casos de testes considerados. Para todos os experimentos, o tamanho da população foi igual a dez vezes o número de geradores (10n) conforme sugerido em [Storn and Price 1995] e o critério de parada adotado foi 2.000.000 de avaliações. Os valores de F e CR foram 0,5 e 0,1, respectivamente ${ }^{1}$. São utilizados três estudos de casos para avaliar a qualidade dos algoritmos propostos: dois com 13 geradores e um com 40 geradores.

\subsection{Resultados para o Primeiro Estudo de Caso - Caso 13 Geradores e Demanda de $2520 \mathrm{MW}$}

O primeiro estudo de caso considera 13 geradores com efeito de ponto de válvula e demanda de $2520 \mathrm{MW}$ [Coelho and Mariani 2006]. A Tabela 1 apresenta os resultados obtidos pelos algoritmos propostos $\left(\mathrm{ED}_{A P}\right.$ e $\left.\mathrm{ED}_{P M}\right)$ e os da literatura.

Para este caso, os algoritmos propostos $\left(\mathrm{ED}_{A P}\right.$ e $\left.\mathrm{ED}_{P M}\right)$, obtiveram os melhores valores para os três indicadores de custo: mínimo $(24164,05)$, médio $(24164,05)$ e máximo $(24164,05)$ com desvio padrão nulo $(0,00)$, indicando que todas as execuções conseguiram atingir o valor mínimo conhecido. Destaca-se que dentre os algoritmos da literatura apenas o SDE consegue achar o valor mínimo, ou seja, considerando todos os

\footnotetext{
${ }^{1}$ Os valores de $\mathrm{F}$ e $\mathrm{CR}$ foram empiricamente determinados.
} 
Algoritmo 1: $\mathrm{ED}_{A P}$ e $\mathrm{ED}_{P M}$

Entrada: Parâmetros NP, CR e F.

Saída: Melhor solução encontrada ao longo da evolução.

início

Inicializar aleatoriamente a população inicial de tamanho NP;

Aplicar o Procedimento de Reparação;

Avaliar cada solução da população inicial;

repita

para cada solução faça

Selecionar o Operador da ED utilizando AP ou PM;

Selecionar M soluções da população;

Aplicar o Operador da ED selecionado;

Aplicar o Procedimento de Reparação;

Avaliar o Custo do novo indivíduo;

Aplicar a Seleção;

Calcular a Recompensa e a Qualidade Empírica;

Atualizar as Probabilidades dos Operadores da ED;

fim

até que o critério de parada seja satisfeito;

fim

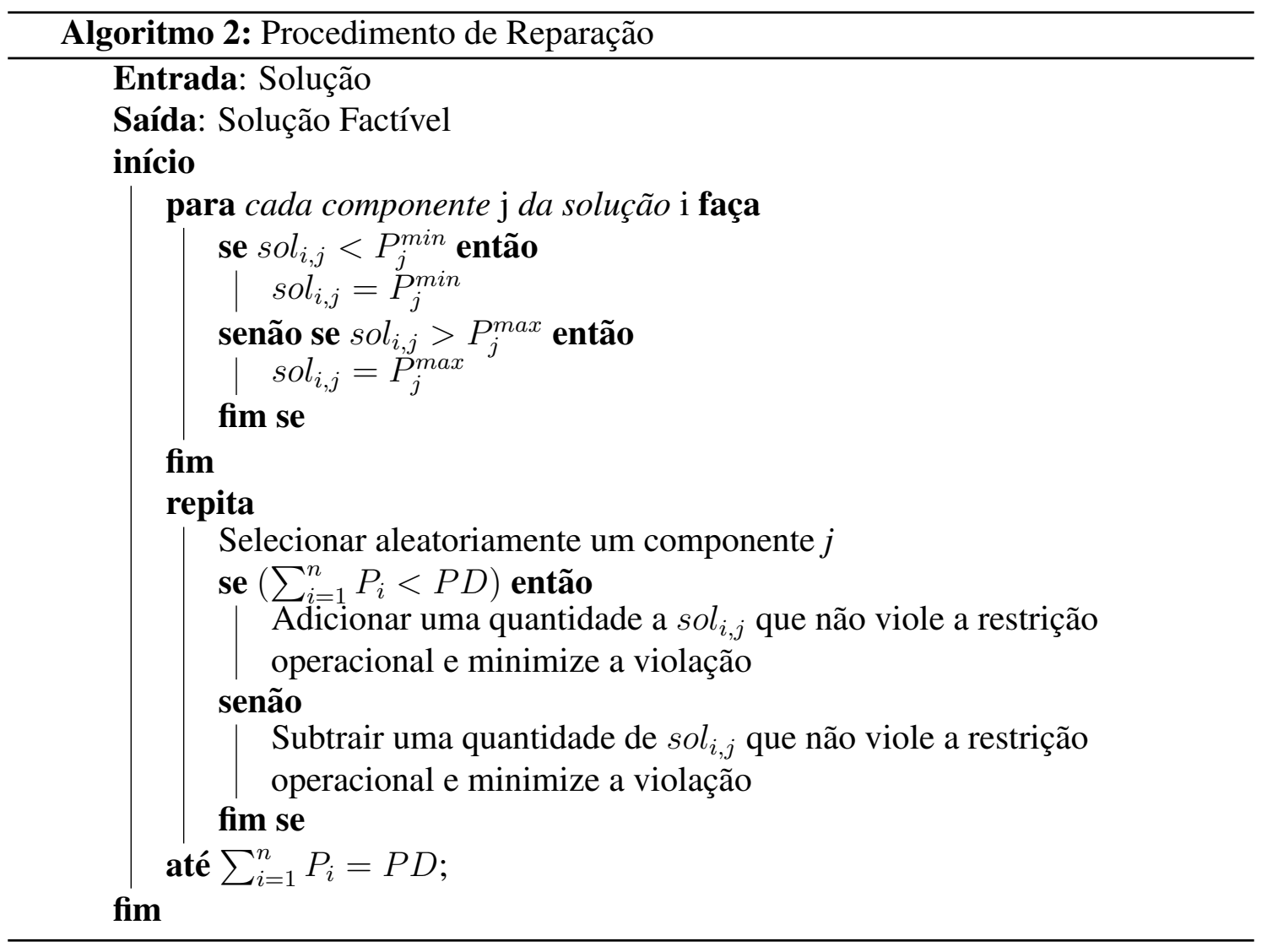


Tabela 1. Resultados para o Primeiro Estudo de Caso. Custos em US\$

\begin{tabular}{l|c|c|c|c}
\hline \hline \multicolumn{5}{c}{ Caso com 13 geradores e demanda 2520 MW } \\
\hline \hline Método & Custo Mín & Custo Médio & Custo Máx & Desvio \\
\hline ED $_{A P}$ & $\mathbf{2 4 1 6 4 , 0 5}$ & $\mathbf{2 4 1 6 4 , 0 5}$ & $\mathbf{2 4 1 6 4 , 0 5}$ & $\mathbf{0 , 0 0}$ \\
\hline ED $_{P M}$ & $\mathbf{2 4 1 6 4 , 0 5}$ & $\mathbf{2 4 1 6 4 , 0 5}$ & $\mathbf{2 4 1 6 4 , 0 5}$ & $\mathbf{0 , 0 0}$ \\
\hline SDE [Balamurugan and Subramanian 2007] & $\mathbf{2 4 1 6 4 , 0 5}$ & 24168,28 & 24200,05 & - \\
\hline DTSA [Khamsawang et al. 2004] & 24169,95 & - & - & - \\
\hline DE [Noman and Iba 2008] & 24169,91 & 24169,91 & 24169,91 & - \\
\hline SSA [Yu and Li 2015] & 24169,91 & 24169,91 & 24169,91 & - \\
\hline DPO [Han et al. 2015] & 24169.90 & 24169.90 & 24169.90 & - \\
\hline HCRO-DE [Roy et al. 2014] & 24164,82 & 24164,98 & 24165,34 & 0.06 \\
\hline TLBO [Banerjee et al. 2015] & 24197,00 & - & - & - \\
\hline ORCSA [Nguyen and Vo 2015] & 24169,91 & - & - & - \\
\hline \hline
\end{tabular}

indicadores de custo os algoritmos propostos tiveram desempenho superior aos reportados na literatura. A Tabela 2 apresenta o melhor resultado obtido pelos algoritmos propostos. A solução é codificada por um vetor $P_{i}, \mathrm{i}=1, \ldots, 13$ com custo mínimo de 24164,05.

Tabela 2. Custo Mínimo para o $1^{\circ}$ Estudo de Caso utilizando $\mathrm{DE}_{A P}$ e $\mathrm{DE}_{P M}$.

\begin{tabular}{llllll}
\hline Potência & Geração & Potência & Geração & Potência & Geração \\
\hline$P_{1}$ & 628,3185 & $P_{6}$ & 159,7331 & $P_{11}$ & 77,3999 \\
$P_{2}$ & 299,1993 & $P_{7}$ & 159,7331 & $P_{12}$ & 92,3998 \\
$P_{3}$ & 294,4846 & $P_{8}$ & 159,7331 & $P_{13}$ & 92,3999 \\
$P_{4}$ & 159,7331 & $P_{9}$ & 159,7329 & $\sum_{j=1}^{13} P_{i}=$ & 2520.00 \\
$P_{5}$ & 159,7331 & $P_{10}$ & 77,3998 & Cost $=$ & 24164,05 \\
\hline
\end{tabular}

\subsection{Resultados para o Segundo Estudo de Caso - 13 Geradores e Demanda de 1800 MW}

O segundo estudo de caso considera o mesmo conjunto de 13 geradores do estudo de caso anterior, mas com demanda de 1800 MW [Coelho and Mariani 2006]. Este problema do despacho econômico de energia possui vários mínimos locais e seu mínimo global é difícil de ser determinado [Coelho et al. 2009].

A Tabela 3 apresenta a comparação dos resutados obtidos pelos algoritmos propostos neste trabalho $\left(\mathrm{ED}_{A P}\right.$ e $\left.\mathrm{ED}_{P M}\right)$ com abordagens recentes da literatura. Neste caso é possível observar que os algoritmos propostos obtiveram melhores valores para o custo mínimo (17960.36) do que as abordagens de comparação. A versão $\mathrm{ED}_{P M}$ obteve o melhor custo médio $(17960,54)$ enquanto o custo médio da versão $\mathrm{ED}_{A P}$ foi melhor do que dos algoritmos reportados na literatura. No quesito custo máximo as abordagens FAPSO-VDE e DPO obtiveram o melhor valor $(17963,83)$. Destaca-se que o custo médio alcançado pelas abordagens propostas é inferior ao custo mínimo reportado por todas as demais abordagens e, portanto, é possível afirmar que as abordagens propostas fazem parte do estado-da-arte para este estudo de caso. A Tabela 4 apresenta o melhor resultado obtido pelos algoritmos propostos codificado por um vetor $P_{i}, \mathrm{i}=1, \ldots, 13$ com custo mínimo de 17960,36.

\subsection{Resultados para o Terceiro Estudo de Caso - 40 Geradores e Demanda de $10500 \mathrm{MW}$}

O Terceiro Estudo de Caso considera 40 geradores com efeito de ponto válvula e demanda de 10500 MW [Ghorbani and Babaei 2016]. O espaço de soluções deste estudo de caso contém múltiplos ótimos locais [Sayah and Hamouda 2013]. 
Tabela 3. Resultados para o Segundo Estudo de Caso. Custos em US\$

\begin{tabular}{l|c|c|c|c}
\hline \hline \multicolumn{5}{c}{ Caso com 13 geradores e demanda de 1800 MW } \\
\hline \hline Método & Custo Mín & Custo Médio & Custo Máx & Desvio \\
\hline ED $_{A P}$ & $\mathbf{1 7 9 6 0 , 3 6}$ & 17961,26 & 17969,34 & 2,72 \\
\hline ED $_{P M}$ & $\mathbf{1 7 9 6 0 , 3 6}$ & $\mathbf{1 7 9 6 0 , 5 4}$ & 17969,34 & $\mathbf{1 , 2 7}$ \\
\hline DEC-SQP [Coelho and Mariani 2006] & 17963,94 & 17973,13 & 17984,81 & 1,97 \\
\hline EP-SQP [Victoire and Jeyakumar 2004] & 17991,03 & 18106,93 & - & - \\
\hline HQPSO5 [Coelho and Mariani 2008] & 17963,95 & 18273,86 & 18633,04 & 123,22 \\
\hline HDE [Wang et al. 2007] & 17975,73 & 18134,80 & - & - \\
\hline ST-HDE [Wang et al. 2007] & 17963,89 & 18046,38 & - & - \\
\hline DE [Noman and Iba 2008] & 17963,83 & 17965,48 & 17975,36 & - \\
\hline CDEMD [Coelho et al. 2009] & 17961,94 & 17974,68 & 18061,41 & 20,30 \\
\hline GSA [Swain et al. 2012] & 17963,84 & 18041,21 & 18910,31 & - \\
\hline FAPSO-VDE [Mohammadi-Ivatloo et al. 2012] & 17963,82 & 17963,82 & $\mathbf{1 7 9 6 3 , 8 3}$ & - \\
\hline FA [Yang et al. 2012] & 17963,83 & 18029,16 & 18168,80 & 148,54 \\
\hline$\theta$-PSO [Hosseinnezhad and Babaei 2013] & 17963,82 & 17965,20 & 17980,20 & 4,38 \\
\hline SSA [Yu and Li 2015] & 17963,76 & - & - & - \\
\hline DPO [Han et al. 2015] & 17963,82 & 17963,82 & $\mathbf{1 7 9 6 3 . 8 3}$ & - \\
\hline TLBO [Banerjee et al. 2015] & 18115,00 & - & - & - \\
\hline ORCSA [Nguyen and Vo 2015] & 17963,83 & - & - & - \\
\hline \hline
\end{tabular}

Tabela 4. Custo Mínimo para o $2^{\circ}$ Estudo de Caso utilizando $\mathrm{DE}_{A P}$ e $\mathrm{DE}_{P M}$.

\begin{tabular}{llllll}
\hline Potência & Geração & Potência & Geração & Potência & Geração \\
\hline$P_{1}$ & 628,3185 & $P_{6}$ & 60 & $P_{11}$ & 40 \\
$P_{2}$ & 149,5996 & $P_{7}$ & 109,8665 & $P_{12}$ & 55 \\
$P_{3}$ & 222,7493 & $P_{8}$ & 109,8665 & $P_{13}$ & 55 \\
$P_{4}$ & 109,8666 & $P_{9}$ & 109,8665 & $\sum_{j=1}^{13} P_{i}=$ & 1800.00 \\
$P_{5}$ & 109,8665 & $P_{10}$ & 40 & Custo = & 17960,36 \\
\hline
\end{tabular}

A Tabela 5 apresenta a comparação entre os resultados obtidos pelo $\mathrm{ED}_{A P} \mathrm{e}$ $\mathrm{ED}_{P M}$ e resultados reportados na literatura recente. $\mathrm{O}$ algoritmo $\mathrm{ED}_{A P}$ obteve o melhor valor para o custo mínimo $(121406,91)$ enquanto o $\mathrm{ED}_{P M}$ obteve os melhores valores para o custo médio $(121411,92)$, custo máximo $(121412,12)$ e desvio padrão $(0,07)$. Destacase que o custo mínimo $(121411,89)$ e o custo médio $(121411,92)$ obtidos pelo $\operatorname{ED}_{P M}$ são inferiores ao menor custo mínimo reportado pelos algoritmos de comparação (121412,53, obtido pelo EMA e ORCSA). Portanto, é possível afirmar que o $\mathrm{ED}_{P M}$ é um algoritmo do estado-da-arte. A Tabela 6 demonstra o melhor resultado obtido com o $\mathrm{ED}_{A P}$ codificado por um vetor $P_{i}, \mathrm{i}=1, \ldots, 40$ com custo igual a 121406,91.

\section{Conclusões e Trabalhos Futuros}

Neste artigo foram investigadas técnicas de seleção adaptativa de operadores da Evolução Diferencial para resolver o problema do Despacho Econômico de Energia Elétrica. As técnicas investigadas foram a Adaptive Pursuit e a Probability Matching e o conjunto de operadores foi composto pela ED/rand/1/bin, ED/rand/2/bin, ED/current-to-rand/1 e ED/current-to-rand/2.

De acordo com os resultados obtidos, os dois métodos implementados $\left(\mathrm{ED}_{A P} \mathrm{e}\right.$ $\mathrm{ED}_{P M}$ ) tiveram bom desempenho nos três casos de teste considerados, obtendo melhores valores que os algoritmos reportados na literatura para o custo mínimo, custo médio e custo máximo em todos os estudos de caso (com exceção do segundo estudo de caso no custo máximo, onde FAPSO-VDE e DPO foram melhores). Destaca-se que o custo médio obtido pelo algoritmo $\mathrm{ED}_{P M}$ em todos os casos é inferior ao menor custo mínimo reportado na literatura e, portanto, é possível concluir que o $\mathrm{ED}_{P M}$ é um algoritmo do estado- 
Tabela 5. Resultados para o Terceiro Estudo de Caso. Custos em US\$

\begin{tabular}{l|c|c|c|c}
\hline \multicolumn{5}{c}{ Caso com 40 geradores e demanda de 10500 MW } \\
\hline \hline Método & Custo Mín & Custo Médio & Custo Máx & Desvio \\
\hline ED $_{A P}$ & $\mathbf{1 2 1 4 0 6 , 9 1}$ & 121414,43 & 121417,51 & 2,37 \\
\hline ED $_{P M}$ & 121411,89 & $\mathbf{1 2 1 4 1 1 , 9 2}$ & $\mathbf{1 2 1 4 1 2 , 1 2}$ & $\mathbf{0 , 0 7}$ \\
\hline CCDE [Ghasemi et al. 2015] & 121412,54 & 121412,99 & 121414,69 & 0,09 \\
\hline EMA [Ghorbani and Babaei 2016] & 121412,53 & 121417,13 & 121426,15 & - \\
\hline HCRO-DE [Roy et al. 2014] & 121412,55 & 121413,11 & 121415,68 & 0.13 \\
\hline SQPSO [Hosseinnezhad et al. 2014] & 121412,57 & 121455,70 & 121709,56 & - \\
\hline ORCSA [Nguyen and Vo 2015] & 121412,53 & 121472,45 & 121596,18 & 0.49 \\
\hline FAPSO-VDE [Mohammadi-Ivatloo et al. 2012] & 121412,56 & 121412,61 & 121412,78 & - \\
\hline SSA [Yu and Li 2015] & 121412,55 & - & - & - \\
\hline QPSO [Meng et al. 2010] & 121448,21 & 122225,07 & - & - \\
\hline NDS [Lin et al. 2011] & 121647,39 & 121647,39 & 121647,39 & - \\
\hline FA [Yang et al. 2012] & 121415,05 & 121416,57 & 121424,56 & 1,78 \\
\hline QGS [Moradi-Dalvand et al. 2012] & 121412,55 & 121423,52 & 121438,68 & - \\
\hline$\theta$-PSO [Hosseinnezhad and Babaei 2013] & 121420,90 & 121509,84 & 121852,42 & 92,39 \\
\hline DEPSO [Sayah and Hamouda 2013] & 121412,56 & 121419,31 & 121468,25 & - \\
\hline DEC-SQP [Coelho and Mariani 2006] & 121741,97 & 122295,12 & 122839,29 & 386,18 \\
\hline NPSO-LRS [Selvakumar and Thanushkodi 2007] & 121664,43 & 122209,31 & 122981,59 & - \\
\hline CEP-PSO [Sinha and Purkayastha 2004] & 123670,00 & 124145,60 & 124900,00 & - \\
\hline PSO [Victoire and Jeyakumar 2004] & 123930,45 & 124154,49 & - & - \\
\hline PSO-SQP [Victoire and Jeyakumar 2004] & 122094,67 & 122245,25 & - & - \\
\hline HDE [Wang et al. 2007] & 121813,26 & 122705,66 & - & - \\
\hline ST-HDE [Wang et al. 2007] & 121698,51 & 122304,30 & - & - \\
\hline DE [Noman and Iba 2008] & 121416,29 & 121422,72 & 121431,47 & - \\
\hline CDEMD [Coelho et al. 2009] & 121423,40 & 121526,73 & 121696,98 & 54,86 \\
\hline CSO [Selvakumar and Thanushkodi 2009] & 121461,67 & 121936,19 & 122844,53 & - \\
\hline BBO [Bhattacharya and Chattopadhyay 2010] & 121426,95 & 121508,03 & 121688,66 & - \\
\hline ICA-PSO [Vlachogiannis and Lee 2009] & 121413,20 & 121428,14 & 121453,56 & - \\
\hline & & & & \\
\hline
\end{tabular}

da-arte para o Problema do Despacho Econômico de Energia Elétrica. Na comparação entre os algoritmos propostos é possível dizer que o $\mathrm{ED}_{P M}$ foi melhor que o $\mathrm{ED}_{A P}$. $\mathrm{O}$ bom desempenho dos algoritmos propostos se deve principalmente à capacidade dos algoritmos propostos em escolher o melhor operador para cada estágio da evolução.

Em trabalhos futuros pretende-se analisar a auto-adaptação dos parâmetros da Evolução Diferencial (F e CR) em conjunto com a seleção adaptativa dos operadores.

\section{Referências}

Balamurugan, R. and Subramanian, S. (2007). Self-adaptive differential evolution based power economic dispatch of generators with valve-point effects and multiple fuel options. International Journal of Electrical, Electronic Science and Engineering.

Banerjee, S., Maity, D., and Kumar, C. (2015). Teaching learning based optimization for economic load dispatch problem considering valve point loading effect. International Journal of Electrical Power and Energy Systems, 73:456-464.

Basu, M. (2002). Fuel constrained economic emission load dispatch using hopfield neural networks. Electric Power Systems Research.

Bhattacharya, A. and Chattopadhyay, P. K. (2010). Biogeography-based optimization for different economic load dispatch problems. IEEE Transactions on Power Systems.

Coelho, L. and Mariani, V. (2008). Particle swarm approach based on quantum mechanics and harmonic oscillator potential well for economic load dispatch with valve-point effects. Energy Conversion and Management, 49(11):1373-1378. 
Tabela 6. Custo Mínimo para o $3^{\circ}$ Estudo de Caso utilizando $\mathrm{DE}_{A P}$.

\begin{tabular}{llll}
\hline Potência & Geração & Potência & Geração \\
\hline$P_{1}$ & 110,8087 & $P_{21}$ & 523,2795 \\
$P_{2}$ & 110,8242 & $P_{22}$ & 523,2880 \\
$P_{3}$ & 97,4105 & $P_{23}$ & 523,2821 \\
$P_{4}$ & 179,7335 & $P_{24}$ & 523,2831 \\
$P_{5}$ & 87,6980 & $P_{25}$ & 523,2890 \\
$P_{6}$ & 139,9999 & $P_{26}$ & 523,2747 \\
$P_{7}$ & 259,6546 & $P_{27}$ & 10,0027 \\
$P_{8}$ & 284,6115 & $P_{28}$ & 10,0000 \\
$P_{9}$ & 284,6543 & $P_{29}$ & 10,0000 \\
$P_{10}$ & 130,0021 & $P_{30}$ & 87,8369 \\
$P_{11}$ & 94,0000 & $P_{31}$ & 189,9998 \\
$P_{12}$ & 94,0000 & $P_{32}$ & 189,9994 \\
$P_{13}$ & 214,7616 & $P_{33}$ & 189,9996 \\
$P_{14}$ & 394,2789 & $P_{34}$ & 164,7942 \\
$P_{15}$ & 394,2789 & $P_{35}$ & 199,9983 \\
$P_{16}$ & 394,2880 & $P_{36}$ & 194,2579 \\
$P_{17}$ & 489,2835 & $P_{37}$ & 110,0000 \\
$P_{18}$ & 489,2768 & $P_{38}$ & 110,0000 \\
$P_{19}$ & 511,2856 & $P_{39}$ & 110,0000 \\
$P_{20}$ & 511,2810 & $P_{40}$ & 511,2817 \\
$\sum_{j=1}^{40} P_{i}=$ & 10500 & Custo $=$ & 121406,62 \\
\hline & & &
\end{tabular}

Coelho, L. S. and Mariani, V. C. (2006). Combining of chaotic differential evolution and quadratic programming for economic dispatch optimization with valve-point effect. IEEE Transactions on Power Systems, 21(2):989-996.

Coelho, L. S., Souza, R. T. T., and Mariani, V. C. (2009). Improved differential evolution approach based on cultural algorithms and diversisty measure applied to solve economic load dispatch problems. Mathematics and Computers in Simulation, 79:31363147.

Engelbrecht, A. P. (2007). Computational Intelligence: An Introduction. Wiley Publishing, 2nd edition.

Ghasemi, M., Taghizadeh, M., Ghavidel, S., and Abbasian, A. (2015). Colonial competitive differential evolution: An experimental study for optimal economic load dispatch. Applied Soft Computing, 40:342-363.

Ghorbani, N. and Babaei, E. (2016). Exchange market algorithm for economic load dispatch. International Journal of Electrical Power and Energy Systems, 75:19-27.

Goldberg, D. E. (1990). Probability matching, the magnitude of reinforcement, and classifier system bidding. Mach. Learn., 5:407-425.

Gong, W., Álvaro Fialho, Cai, Z., and Li, H. (2011). Adaptive strategy selection in differential evolution for numerical optimization: An empirical study. Inform. Sciences, 181(24):5364 - 5386.

Gong, W., Fialho, A., and Cai, Z. (2010). Adaptive strategy selection in differential evolution. In Conference on Genetic and Evolutionary Computation, pages 409-416.

Han, L., Romero, C. E., and Yao, Z. (2015). Economic dispatch optimization algorithm based on particle diffusion. Energy Conversion and Management, 105:1251-1260.

Hosseinnezhad, V. and Babaei, E. (2013). Economic load dispatch using $\theta$-pso. Energy Conversion and Management, 49:160 - 169. 
Hosseinnezhad, V., Rafiee, M., Ahmadian, M., and Ameli, M. T. (2014). Species-based Quantum Particle Swarm Optimization for economic load dispatch. International Journal of Electrical Power \& Energy Systems, 63:311-322.

Khamsawang, S., Pothiya, S., and Boonseng, S. (2004). Distributed tabu search algorithm for solving the economic dispatch problem. In TENCON, pages 484-487, Chiang Mai, Thailand. TENCON.

Li, K., Fialho, A., and Kwong, S. (2011). Multi-objective differential evolution with adaptive control of parameters and operators. In International Conference on Learning and Intelligent Optimization, pages 473-487.

Lin, W.-M., Gow, H.-J., and Tsai, M.-T. (2011). Combining of direct search and signal-tonoise ratio for economic dispatch optimization. Energy Conversion and Management, 52(1):487 - 493 .

Mallipeddi, R., Suganthan, P., Pan, Q., and Tasgetiren, M. (2011). Differential evolution algorithm with ensemble of parameters and mutation strategies. Appl. Soft Comput., 11(2):1679-1696.

Meng, K., Wang, H. G., Dong, Z., and Wong, K. P. (2010). Quantum-inspired particle swarm optimization for valve-point economic load dispatch. IEEE Transactions on Power Systems, 25(1):215-222.

Mohammadi-Ivatloo, B., Rabiee, A., Soroudi, A., and Ehsan, M. (2012). Iteration $\{$ PSO with time varying acceleration coefficients for solving non-convex economic dispatch problems. International Journal of Electrical Power \& Energy Systems, 42(1):508 516.

Moradi-Dalvand, M., Mohammadi-Ivatloo, B., Najafi, A., and Rabiee, A. (2012). Continuous quick group search optimizer for solving non-convex economic dispatch problems. Electric Power Systems Research, 93(0):93 - 105.

Nguyen, T. T. and Vo, D. N. (2015). The application of one rank cuckoo search algorithm for solving economic load dispatch problems. Applied Soft Computing Journal, 37:763-773.

Noman, N. and Iba, H. (2008). Differential evolution for economic load dispatch problems. Electric Power Systems Research, 78(3):1322-1331.

Park, J., Jeong, Y., and Lee, W. (2006). An improved particle swarm optimization for economic dispatch problems with non-smooth cost functions. In IEEE Power Engineering Society General Meeting, Montreal, Que.

Qin, A. K., Huang, V. L., and Suganthan, P. N. (2009). Differential evolution algorithm with strategy adaptation for global numerical optimization. IEEE Trans. Evol. Comput., 13:398-417.

Qin, A. K. and Suganthan, P. N. (2005). Self-adaptive differential evolution algorithm for numerical optimization. In IEEE Congress on Evolutionary Computation, pages $1785-1791$.

Roy, P. K., Bhui, S., and Paul, C. (2014). Solution of economic load dispatch using hybrid chemical reaction optimization approach. Applied Soft Computing, 24:109-125. 
Sayah, S. and Hamouda, A. (2013). A hybrid differential evolution algorithm based on particle swarm optimization for nonconvex economic dispatch problems. Applied Soft Computing, 13(4):1608 - 1619.

Selvakumar, A. and Thanushkodi, K. (2009). Optimization using civilized swarm: solution to economic dispatch with multiple minima. Electric Power Systems Research, 79(1):8-16.

Selvakumar, A. I. and Thanushkodi, K. (2007). New particle swarm optimization solution to nonconvex economic dispatch problems. IEEE Transactions on Power Systems, 22(1):42-51.

Sinha, N. and Purkayastha, B. (2004). Pso embedded evolutionary programming technique for nonconvex economic load dispatch. In IEEE PES - Power Systems Conference and Exposition, pages 66-71, Brasil. IEEE PES.

Storn, R. and Price, K. (1995). Differential evolution - a simple and efficient adaptive scheme for global optimization over continuous spaces.

Storn, R. and Price, K. (1997). Differential evolution - a simple and efficient heuristic for global optimization over continuous spaces. J. Global Optim., 11(4):341-359.

Swain, R., Sahu, N., and Hota, P. (2012). Gravitational search algorithm for optimal economic dispatch. Procedia Technology, 6(0):411 - 419.

Thierens, D. (2005). An adaptive pursuit strategy for allocating operator probabilities. In Conference on Genetic and Evolutionary Computation, pages 1539-1546.

Victoire, T. and Jeyakumar, A. (2004). Hybrid pso-sqp for economic dispatch with valvepoint effect. Electric Power Systems Research, 71(1):51-59.

Vlachogiannis, J. G. and Lee, K. Y. (2009). Economic load dispatch - a comparative study on heuristic optimization techniques with an improved coordinated aggregation-based pso. IEEE Transactions on Power Systems, 24(2):991-1001.

Wang, S. K., Chiou, J. P., and Liu, C. W. (2007). Non-smooth/nonconvex economic dispatch by a novel hybrid differential evolution algorithm. IET Generation, Transmission \& Distribution, 1(5):793-803.

Yang, X.-S., Hosseini, S. S. S., and Gandomi, A. H. (2012). Firefly algorithm for solving non-convex economic dispatch problems with valve loading effect. Applied Soft Computing, 12(3):1180 - 1186.

Yu, J. J. Q. and Li, V. O. K. (2015). A social spider algorithm for solving the non-convex economic load dispatch problem. Neurocomputing, 171:1-11.

Zhang, J. and Sanderson, A. C. (2009). JADE: adaptive differential evolution with optional external archive. IEEE Trans. Evol. Comput., 13:945-958.

Zhao, S.-Z., Suganthan, P. N., and Zhang, Q. (2012). Decomposition-based multiobjective evolutionary algorithm with an ensemble of neighborhood sizes. IEEE Trans. Evol. Comput., 16(3):442-446. 